\title{
Neurologically normal survival after fibrinolysis during prolonged cardiac arrest: case report and discussion
}

\author{
Kevin Clark, MD; ${ }^{*}$ Riyad B. Abu-Laban, MD, MHSc; ${ }^{\dagger}$ Peter J. Zed, BSc (Pharm), PharmD; ${ }^{\dagger \dagger}$ \\ Lois Graham, $\mathrm{MD}^{\dagger}$
}

\begin{abstract}
Cardiac arrest secondary to pulmonary embolism is a devastating condition with a high mortality rate. It is currently unclear whether fibrinolysis (thrombolysis) is beneficial in this setting. We report the case of a 28-year-old woman with a pulmonary embolism who developed return of pulses following the administration of tissue plasminogen activator after 38 minutes of pulseless electrical activity cardiac arrest. She went on to make a full neurologic and cardiopulmonary recovery. This case is discussed with reference to the current literature on the subject.

Key words: cardiac arrest, fibrinolysis, thrombolysis, pulmonary embolism

RÉSUMÉ

L'arrêt cardiaque découlant de l'embolie pulmonaire est une affection dévastatrice associée à un taux élevé de mortalité. Actuellement, les bienfaits de la fibrinolyse (thrombolyse) n'ont pas été déterminés avec certitude dans un tel contexte. Nous présentons le cas d'une femme âgée de 28 ans atteinte d'une embolie pulmonaire dont le pouls est revenu après l'administration d'un activateur tissulaire du plasminogène. Elle avait été en arrêt cardiaque avec activité électrique sans pouls pendant 38 minutes. Elle a par la suite connu une récupération neurologique et cardio-pulmonaire complète. Ce cas est discuté dans le cadre de la littérature présentement disponible sur le sujet.
\end{abstract}

\section{Introduction}

Cardiac arrest secondary to pulmonary embolism (PE) is a devastating condition with a high mortality rate. ${ }^{1}$ The Advanced Cardiac Life Support (ACLS) guidelines consider PE a "potentially reversible" cause of cardiac arrest ${ }^{2}$ however, whether fibrinolysis (thrombolysis) in such situations is advocated while cardiopulmonary resuscitation (CPR) is ongoing, particularly when the diagnosis of PE is presumptive, is not clear.

We report the case of a 28-year-old woman who appeared to respond to tissue plasminogen activator (t-PA) given during CPR for the presumptive, and subsequently proven, diagnosis of PE. After 38 minutes of pulseless electrical activity (PEA) cardiac arrest, our patient developed return of spontaneous circulation (ROSC, defined as the return of a palpable pulse) shortly after t-PA was administered. She went on to make a full neurological and cardiopulmonary recovery. This case is discussed with reference to the current literature on the subject.

\section{Case report}

A 28-year-old woman was transported to the emergency department (ED) 30 minutes after collapsing at work. On arrival she was pale and distressed with grunting respirations. She had a heart rate of 37 beats/min, a respiratory rate of 24 breaths/min, a blood pressure of $58 / 0 \mathrm{~mm} \mathrm{Hg}$, and a Glas-

*University of British Columbia FRCP Emergency Medicine Residency Program, Vancouver, BC

tDepartment of Emergency Medicine, Vancouver General Hospital, and Division of Emergency Medicine, University of British Columbia $\neq$ CSU Pharmaceutical Sciences, Vancouver General Hospital, and Faculty of Pharmaceutical Sciences, Vancouver General Hospital, University of British Columbia, Vancouver, BC

Received: Aug. 12, 2002; final submission: Nov. 14, 2002; accepted: Nov. 14, 2002

This article has been peer reviewed. 
gow Coma Scale (GCS) score of 11. Her peripheral perfusion was poor, and her oxygen saturation by pulse oximetry was $90 \%$ on $100 \%$ oxygen. Jugular venous distension was present, and auscultation revealed clear air entry bilaterally, normal heart sounds and no murmurs. Her abdomen was not tender or distended and she had no evidence of a deep vein thrombosis. Her pupils were $4 \mathrm{~mm}$ and reactive, and the remainder of the physical examination was unremarkable.

The patient's history was significant for an arthroscopic anterior cruciate ligament and meniscal repair of the left knee 2 weeks prior to presentation. She had been using crutches since the surgery, and her only medications were ibuprofen and naproxen. She had previously been on an oral contraceptive agent but had discontinued this 3 months prior to her surgery.

The bradycardia resolved, however, persisting hemodynamic instability and hypoxia mandated the decision to proceed with endotracheal intubation within minutes of the patient's arrival. Rapid sequence intubation was performed with intravenous (IV) etomidate $(20 \mathrm{mg}$ ) and succinylcholine (120 mg). During intubation the patient's heart rate dropped to 35 beats/min and two 1-mg doses of IV atropine were administered. Endotracheal tube placement was confirmed by capnography, which showed an appropriate waveform and end tidal $\mathrm{CO}_{2}$ level of $26 \mathrm{~mm} \mathrm{Hg}$.

Immediately following intubation the patient lost pulses (confirmed by multiple health care providers) and was found to be in narrow complex PEA at a rate of 150 beats/min. Cardiopulmonary resuscitation was commenced, and epinephrine $1 \mathrm{mg}$ IV resulted in a transient ROSC with palpable pulses for 2 minutes. Pulseless electrical activity then returned, CPR was recommenced, and interventions following the ACLS guidelines for PEA were performed, including the administration of IV fluids, and repeated 1-mg doses of epinephrine every 3 to 5 minutes.

The two conditions considered to be most likely as a cause of the patient's presentation were ruptured ectopic pregnancy (or similar intra-abdominal catastrophe) or massive PE. Stat requests were made for a transthoracic echocardiogram and an abdominal ultrasound. During these investigations, the patient remained in narrow complex PEA at a rate of 150 beats/min and CPR was continued. A chest radiograph was unremarkable, and an arterial blood gas showed the following: $\mathrm{pH}$ 6.95; $\mathrm{PCO}_{2} 37 ; \mathrm{PO}_{2} 337$; and bicarbonate 8. A urine pregnancy test was negative. The abdominal ultrasound was limited, but showed no evidence of free intraperitoneal fluid. The transthoracic echocardiogram showed an enlarged right ventricle with severe hypokinesis, paradoxical motion of the interventricular septum, severe tricuspid regurgitation and poor left ventricular function (Fig. 1).
At this point, it was felt that the patient almost certainly had a massive PE. The decision was made to proceed with fibrinolysis while CPR was ongoing. Thus, 29 minutes after the patient's arrival in the ED and 22 minutes after CPR was started for PEA, IV t-PA was commenced. Initially, a 15 -mg bolus was administered following the standard acute myocardial infarction protocol (a 15-mg bolus followed by 2 infusions over a period of 90 minutes). This protocol was subsequently altered, and a further $85 \mathrm{mg}$ was infused over 1 hour. A norepinephrine infusion was also initiated shortly after the t-PA was commenced.

Sixteen minutes after the t-PA bolus, and 38 minutes after the onset of continuous CPR, ROSC occurred. Initial vital signs were blood pressure 108/63 $\mathrm{mm} \mathrm{Hg}$, heart rate 140 beats/min and oxygen saturation by pulse oximetry $99 \%$. During the cardiac arrest, the patient had received 40 $\mathrm{mg}$ of t-PA, $10 \mathrm{mg}$ of epinephrine, $1 \mathrm{gm}$ of $10 \%$ calcium chloride and $50 \mathrm{mEq}$ of sodium bicarbonate, all IV. An ECG after ROSC occurred revealed sinus tachycardia, prominent $\mathrm{S}$ waves in lead I, and inverted $\mathrm{T}$ waves in lead III (Fig. 2). The patient was subsequently transferred to the intensive care unit on a unfractionated heparin infusion.

Less than 24 hours after intensive care unit admission, the patient was extubated. A spiral CT of the chest showed bilateral pulmonary emboli with an infarcted right lung base. A compression ultrasound showed an extensive deep vein thrombosis of the left (post-operative) leg. Four days later a transthoracic echocardiogram showed trivial tricuspid regurgitation, normal biventricular function and normal

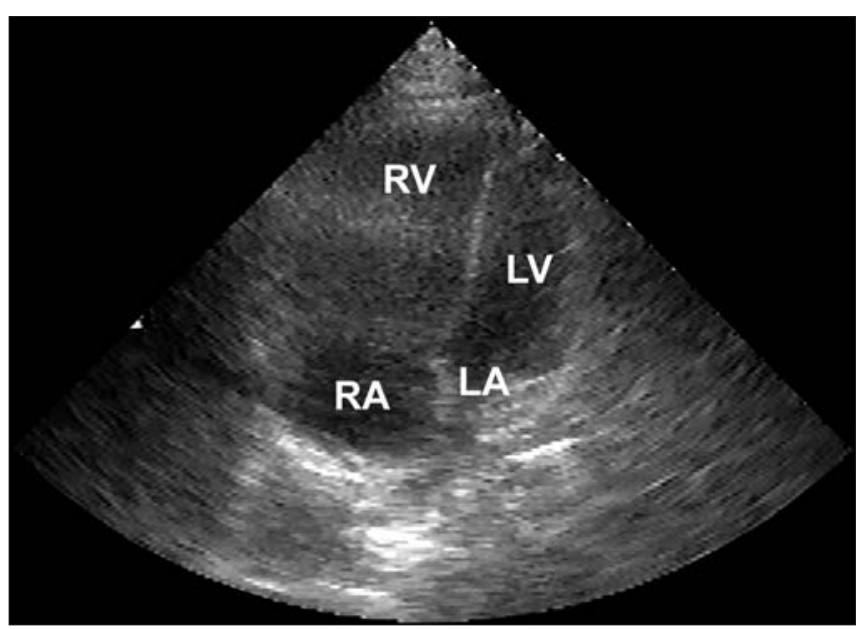

Fig. 1. Still image from the transthoracic echocardiogram taken while CPR was ongoing. This image shows an enlarged right ventricle (RV) with paradoxical motion of the interventricular septum into the left ventricle (LV). The left atrium (LA) and right atrium (RA) are visible. Severe right ventricular hypokinesis, severe tricuspid regurgitation, and poor left ventricular function were also evident during the study. 
pulmonary artery pressures (Fig. 3). An abdominal CT showed a hepatic hematoma, however the unfractionated heparin was continued. The patient then developed heparin-induced thrombocytopenia necessitating substitution of danaproid for anticoagulation.

The patient was discharged from hospital on oral warfarin 19 days after presentation, and subsequent hematologic investigations revealed the presence of a prothrombin gene mutation predisposing to thrombosis. At the time of discharge, the patient had normal cardiopulmonary function and no functional or cognitive neurologic deficits.

\section{Discussion}

Pulmonary embolism is reported to cause 200000 deaths annually in the United States, one-third of which occur in the first hour from symptom onset. ${ }^{3}$ Historically, PE significant enough to cause hemodynamic instability was treated with surgical embolectomy. Fibrinolytic agents are now increasingly advocated as first-line therapy for such situations, ${ }^{1,4}$ particularly when evidence of right ventricular dysfunction is present. ${ }^{5}$ Although prospective randomized trials on fibrinolysis in unstable PE have been lacking, this therapy has become more common because it has been shown to have an early effect on right ventricular function and pulmonary perfusion. ${ }^{6}$ Recently, a randomized double-blind trial of heparin and t-PA versus heparin and placebo for patients with acute submassive PE was published with an accompanying editorial. ${ }^{7,8}$

This study concluded that t-PA, in conjunction with he- parin, can improve the clinical course of patients with echocardiographic evidence of right ventricular dsyfunction and normal systemic blood pressure, and prevent deterioration requiring treatment escalation during the hospital stay.

Although cardiac arrest from PE can present with any rhythm, it is clear that PEA is most highly associated with this condition. ${ }^{9,10}$ Pulseless electrical activity comprises $20 \%$ of cardiac arrests and has a survival-to-hospital discharge rate of approximately $4 \% .{ }^{11}$ Most PEA survivors re-

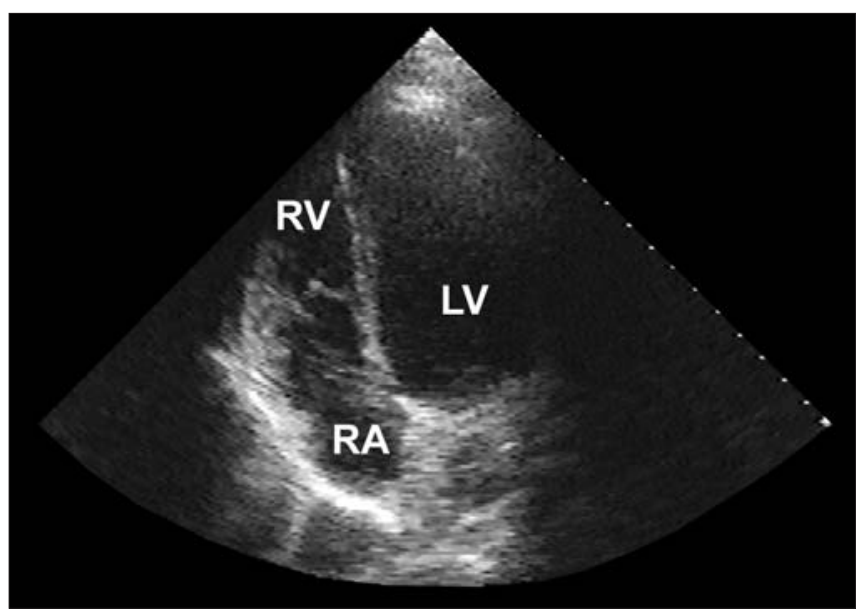

Fig. 3. Still image from the transesophageal echocardiogram taken 4 days after ROSC occurred. This image shows resolution of the abnormalities in Fig. 1. The right ventricle (RV), left ventricle (LV) and right atrium (RA) all appear normal, as does the interventricular septum. Persisting trivial tricuspid regurgitation, normal biventricular function and normal pulmonary artery pressures were also evident during this study.

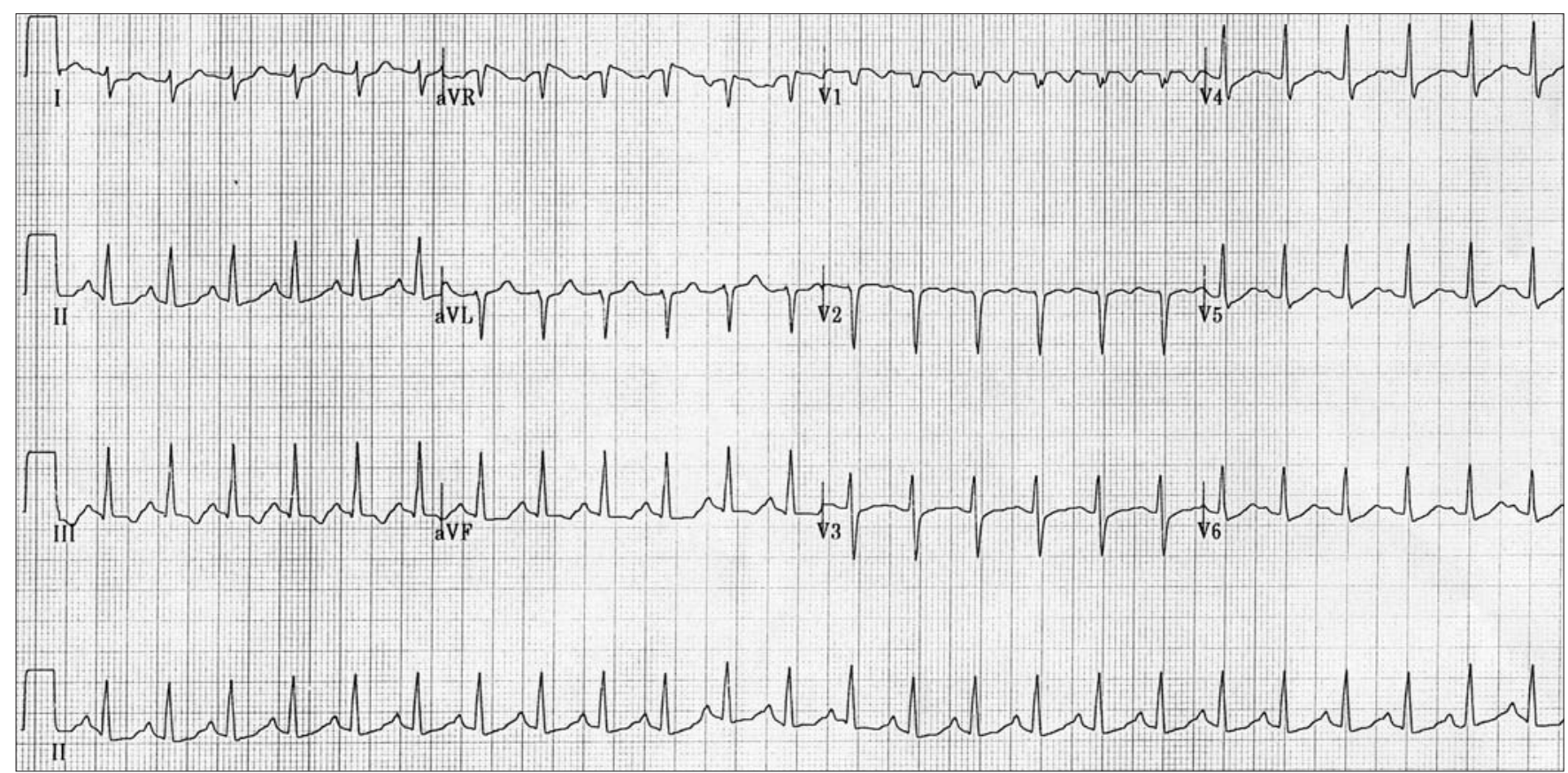

Fig. 2. ECG taken after ROSC occurred showing prominent S waves in lead I and inverted T waves in lead III. 
spond to initial interventions early during resuscitative efforts, ${ }^{11,12}$ and, although poorly studied, this dismal outcome might not be consistent across all subgroups, particularly patients with PE. PEA is unique among cardiac arrest rhythms in that forward blood flow can occur. ${ }^{13-15}$ This has prompted heightened vigilance for reversible causes when treating sustained PEA. ${ }^{16}$ The possibility of low-grade cerebral and coronary perfusion during PEA supports the concept that a good outcome can result even with prolonged cardiac arrest if such a cause is addressed.

There are no validated criteria to guide physicians in selecting a subgroup of cardiac arrest patients likely to have a $\mathrm{PE}$, although it seems intuitive that limb immobilization or recent surgery (both of which our patient had), or a known pulmonary embolism or deep vein thrombosis, would define a high-risk group. Bedside imaging, in centres such as ours where it is available, may also be helpful. Although the role of abdominal ultrasound in cardiac arrest is undefined, it again seems intuitive that lack of ultrasound evidence of intra-abdominal bleeding, in a patient for whom this is considered a potential etiology, would provide increased support for the diagnosis of PE. A number of case reports have suggested that echocardiography during cardiac arrest can be helpful in establishing the diagnosis of $\mathrm{PE}, 5$ and the results of this investigation along with an abdominal ultrasound influenced the decision to administer t-PA to our patient.

In 1989, a case report was published in the Annals of Emergency Medicine describing the administration of t-PA during cardiac arrest for presumed, and subsequently proven, PE. ${ }^{17}$ This case was remarkably similar to the one we present in that it described a 33-year-old woman who collapsed 1 week after an abdominal hysterectomy, had 30 minutes of PEA, developed ROSC shortly after receiving t-PA, and went on to make a full neurologic recovery. A number of similar cases have been reported, many in European journals. ${ }^{18-23}$ Our case adds to this literature and is the first such report from a Canadian centre. A review in 2000 summarized 9 papers describing 67 patients treated with fibrinolytic agents during cardiac arrest. ${ }^{24}$ The majority of patients had known or suspected $\mathrm{PE}$ and the overall survival was $75 \%$. Only 3 survivors had documented neurologic sequelae, despite an overall mean arrest duration of 51 minutes.

The evidence cited above for the use of fibrinolysis in PEinduced cardiac arrest is weak, and predominantly in the form of case reports. Collectively, however, this literature presents a compelling argument for a potential beneficial effect from such therapy, and certainly supports the concept that complete recovery can result despite prolonged PEA. Unfortunately, it is impossible to know how many times this therapy has been administered unsuccessfully and what im- pact "reporting bias" plays in the predominately positive published cases. We suspect many emergency physicians have, on occasion, administered a fibrinolytic agent while CPR was ongoing and not reported the outcomes.

To date there have been no rigorous studies of fibrinolysis during cardiac arrest known, or highly suspected, to be induced by PE. Two methodologically weak studies have suggested a benefit from t-PA in a general cardiac arrest population, ${ }^{25,26}$ however, the only randomized controlled trial of such an approach was negative. ${ }^{27}$ In this recently published Canadian trial, the "t-PA in PEA Study," 233 undifferentiated cardiac arrest patients in Greater Vancouver (those whose arrest etiology was unknown or presumed to be cardiovascular) with at least 1 minute of PEA were randomized to receive double-blind t-PA $(100 \mathrm{mg})$ or placebo as a 15-minute infusion while CPR was ongoing, followed by at least 15 further minutes of resuscitative efforts. This study found no evidence of an increased rate of ROSC from t-PA and had one survivor to discharge in the t-PA arm $(0.9 \%)$ and none in the placebo arm $(p=0.99)$. The 95\% confidence intervals excluded a t-PA-related increase in survival-to-hospital discharge of over $4.8 \%$.

The challenge emergency physicians face when confronted with a patient in whom the diagnosis of PE-induced cardiac arrest is certain or highly suspected, such as in the case we present, is that it is unclear from the literature whether fibrinolytic administration may be beneficial. For a study to answer this question, it would have to be multicentre and either enroll an extremely large sample or utilize a modality, such as transthoracic or transesophageal echocardiography ${ }^{13,14}$ or a decision rule ${ }^{10}$ to enroll a population with a high probability of PE. It is clear from the "t-PA in PEA Study" that there is minimal, if any, benefit from t-PA in an undifferentiated PEA population. ${ }^{27}$ However, the authors of this study concluded that it remains unknown whether a lesser, clinically relevant, treatment effect exists or whether selected subgroups might benefit from this therapy - in particular, those with known or suspected PE or myocardial infarction who meet criteria for a fibrinolytic agent. ${ }^{28} \mathrm{~A}$ clinical trial is currently ongoing in Europe that may shed further light on this issue. ${ }^{29,30}$ If a fibrinolytic is administered, it is also unknown what agent and what administration protocol to use because the pharmacokinetics of these drugs in cardiac arrest are poorly understood. An argument can be made for using t-PA in such a situation, and many of the reported cases have used this drug; however, newer agents deliverable by bolus may be preferable. The suggested dose of t-PA for unstable PE is $100 \mathrm{mg}$ over 2 hours, ${ }^{1,4}$ however, in the arrest situation a more rapid administration, such as the 15-minute infusion used in the "t-PA in PEA Study," would seem warranted. 


\section{Conclusions}

This case provides further suggestive evidence that fibrinolysis may benefit selected patients with PE-induced cardiac arrest. Given the limitations of the current literature, both in facilitating the selection of arrested patients likely to have a PE and in addressing the question of fibrinolytic efficacy, either administering or not administering a fibrinolytic are reasonable actions to take in such a situation.

Our own approach would be to err on the side of treatment in patients who are known or highly suspected to have PE-induced cardiac arrest, particularly if other encouraging signs are present such as narrow QRS complexes, significant levels of end-tidal $\mathrm{CO}_{2}$, evidence of forward blood flow by Doppler or invasive monitoring, or evidence of cardiac activity by echocardiography.

\section{Competing interests: None declared}

Acknowledgements: We thank Dr. David W. Harrison for the important role he played in the genesis of this publication, and Dr. John Jue for providing us with the echocardiogram images.

Dr. Riyad B. Abu-Laban is supported by a Clinical Scholar award from the Michael Smith Foundation for Health Research.

\section{References}

1. Wood KE. Major pulmonary embolism: review of a pathophysiologic approach to the golden hour of hemodynamically significant pulmonary embolism. Chest 2002;121:877-905.

2. 7C: A guide to the international ACLS algorithms. Circulation 2000;102(suppl I):142-57.

3. Schoenfeld CN. Pulmonary embolism. In: Tintinalli JE, Kelen GD, Stapczynski JS, editors. Emergency medicine: a comprehensive study guide. New York: McGraw Hill; 2000. p. 396-401.

4. Hyers TM, Agnelli G, Hull R, Morris TA, Samama M, Tapson $\mathrm{V}$, et al. Antithrombotic therapy for venous thromboembolic disease. Chest 2001;119(suppl):176S-93S.

5. MacCarthy P, Worrall A, McCarthy G, Davies J. The use of transthoracic echocardiography to guide thrombolytic therapy during cardiac arrest due to massive pulmonary embolism. Emerg Med J 2002;19:178-9.

6. Goldhaber SZ, Haire WD, Feldstein ML, Miller M, Toltzis R, Smith JL, et al. Alteplase versus heparin in acute pulmonary embolism: randomized trial assessing right-ventricular function and pulmonary perfusion. Lancet 1993;341:507-11.

7. Konstantinides S, Geibel A, Heusel G, Heinrich R, Kasper W. Heparin plus alteplase compared with heparin alone in patients with submassive pulmonary embolism. N Engl J Med 2002;347 (15):1143-50.

8. Goldhaber SZ. Thrombolysis for pulmonary embolism. N Engl J Med 2002;347(15):1131-2.

9. Kurkciyan I, Meron G, Sterz F, Janata K, Domanovits H, Holzer $\mathrm{M}$, et al. Pulmonary embolism as cause of cardiac arrest: presentation and outcome. Arch Intern Med 2000;160:1529-35.

10. Courtney DM, Sasser HC, Pincus CL, Kline JA. Pulseless electrical activity with witnessed arrest as a predictor of sudden death from massive pulmonary embolism in outpatients. Resuscitation 2001:49:265-72.

11. Stueven HA, Aufderheide T, Waite EM, Mateer JR. Electromechanical dissociation: Six years prehospital experience. Re- suscitation 1989;17:173-82.

12. Bonnin MJ, Pepe PE, Kimball KT, Clark PS. Distinct criteria for termination of resuscitation in the out-of-hospital setting. JAMA 1993;270:1457-62.

13. van der Wouw PA, Koster RW, Delemarre BJ, de Vos R, Lampe-Schoenmaeckers AJ, Lie KI. Diagnostic accuracy of transesophageal echocardiography during cardiopulmonary resuscitation. J Am Coll Cardiol 1997;30:780-3.

14. Varriale P, Maldonado JM. Echocardiographic observations during inhospital cardiopulmonary resuscitation. Crit Care Med 1997; 1997:10.

15. Calinas-Correia J, Phair I. Is there a pulse? Resuscitation 1999; 41:201-2.

16. Cripps T, Camm J. The management of electromechanical dissociation. Resuscitation 1991;22:173-80.

17. Langdon RW, Swicegood WR, Schwartz DA. Thrombolytic therapy of massive pulmonary embolism during prolonged cardiac arrest using recombinant tissue-type plasminogen activator. Ann Emerg Med 1989;18(6):678-80.

18. Kohle W. [Thrombolysis in resuscitated patients with pulmonary embolism.] Anaesthesist 1984;33:469.

19. Scholz KH, Hilmer T, Schuster S, Wojcik J, Kreuzer H, Tebbe U. Thrombolyse bei reanimierten patienten mit lungenembolie (German). Dtsch Med Wschr 1990;115:930-5.

20. Westhoff-Bleck M. Lysetherapie bie protraheirter kardio-pulmonaler reanimation: nutzen und komplikationen. Z Kardiol 1991;80:139. [137 to 143 ]

21. Bottiger BW, Reim SM, Diezel G, Bohrer H, Martin E. High-dose bolus injection of urokinase: use during cardiopulmonary resuscitation for massive pulmonary embolism. Chest 1994;106:1281-3.

22. Pharo GH, Andonakakis A, Chandrasekaren K, Amron G, Levitt JD. Survival from catastrophic intraoperative pulmonary embolism. Anesth Analg 1995;81:188-90.

23. Schulte-Sinkus D, Standl T. [Successful resuscitation after bolus injection of tissue-type plasminogen activator in emergency admission.] Anasthesiol Intensivmed Notfallmed Schmerzther 1998;33:124-8.

24. Newman DH, Greenwald I, Callaway CW. Cardiac arrest and the role of thrombolytic agents. Ann Emerg Med 2000;35:472-0.

25. Bottiger BW, Bode C, Kern S, Gries A, Gust R, Glatzer R, et al. Efficacy and safety of thrombolytic therapy after initially unsuccessful cardiopulmonary resuscitation: a prospective clinical trial. Lancet 2001;357:1583-5.

26. Lederer W, Lichtenberger C, Pechlaner C, Kroesen G, Baubin M. Recombinant tissue plasminogen activator during cardiopulmonary resuscitation in 108 patients with out-of-hospital cardiac arrest. Resuscitation 2001;50:71-6.

27. Abu-Laban RB, Christenson JM, Innes GD, van Beek CA, Wanger KP, McKnight RD, et al. Tissue plasminogen activator in cardiac arrest with pulseless electrical activity. N Engl J Med 2002;346:1522-8.

28. Tiffany PA, Schultz M, Steuven H. Bolus thrombolytic infusions during CPR for patients with refractory arrest rhythms: outcome of a case series. Ann Emerg Med 1998;31:124-6.

29. Bottiger B, Padosch SA, Wenzel V. Tissue plasminogen activator in cardiac arrest with pulseless electrical activity [letter]. N Engl J Med 2002;347:1281.

30. Abu-Laban RB, Christenson JM, Innes GD. Tissue plasminogen activator in cardiac arrest with pulseless electrical activity [letter]. N Engl J Med 2002;347:1282.

Correspondence to: Dr. Riyad B. Abu-Laban, Department of Emergency Medicine, Vancouver General Hospital, 855 W. 12th. Ave., Vancouver BC V5Z 1M9; 604 875-4700, fax 604 875-4872, abulaban@interchange.ubc.ca 